\title{
Fabrication of Polyimide Optical Waveguide on Silicon Dioxide Layer Stacked Silicon Substrate
}

\author{
Dadin Mahmudin*, Shobih, Pamungkas Daud, Yusuf Nur Wijayanto \\ Research Center for Electronics and Telecommunication (PPET) \\ Indonesian Institute of Sciences (LIPI) \\ Komp. LIPI Gd 20, Jl Sangkuriang 21/54D - 40135 \\ Bandung, Indonesia
}

\begin{abstract}
Optical waveguides are important for guiding lightwave from a place to other places. Propagation and insertion losses of the optical waveguides should be considered to be in low values. Recently, optical waveguides with circular structures, which are optical fibers, are used widely for guiding lightwave in long-distance optical communication with very low propagation and insertion losses. Simultaneously, optical waveguides with planar structure are also developed for short distance communication in optical devices. We have reported design and analysis of the planar optical waveguides. In this paper, fabrication of planar optical waveguides using a polyimide material on thin silicon dioxide combined with the silicon substrate is reported. The polyimide material is used for the core of the optical waveguides. The silicon dioxide located on the silicon substrate and the air is used for cladding of the optical waveguides. Fabrication of the optical waveguides such as oxidation, photoresist coating, masking, ultra-violet exposure, and etching was done. The fabricated optical waveguides were characterized physically using a standard microscope and scanning electron microscope (SEM). The fabrication processes and characterization results are reported and discussed in detail.
\end{abstract}

Keywords: optical waveguide, polyimide, silicon dioxide, silicon.

\section{INTRODUCTION}

High-speed communication is required in the future for long and short distance. One of the promising methods is using the light wave as a carrier. It can be propagated through long optical fiber installed under the sea for communication backbone and through the short optical waveguide in an integrated circuit for optical interconnect [1]-[2]. Integrated optics concern on creating simple and compact optical devices on a chip with small size. The integrated optic devices are important to optical interconnect for high-speed communications with very low transmission loss. These devices also benefit from being immune to electromagnetic interference. Integrated optical components interface with such fibers to form devices such as Mach-Zender interferometers, amplitude, and phase modulators, as well as ring resonators for digital filtering [3]-[4].

Figure 1 shows the typical optical interconnect for an optical transmitter developed by Fujitsu Laboratories Limited [5]. The development of an integrated silicon optical transmitter for use in an optical transceiver was announced, which is essential for enabling large volumes of data to be transmitted between Control Processing Unit (CPU). The prototype optical transmitter consists of several optical components such as light source, photodetector, multiplexer,

\footnotetext{
* Corresponding Author.

Email: dadin.mahmudin@lipi.go.id

Received: August 8, 2017 ; Revised: November 3, 2017

Accepted: November 6, 2017 ; Published: December 30, 2017

(C) 2017 PPET - LIPI
}

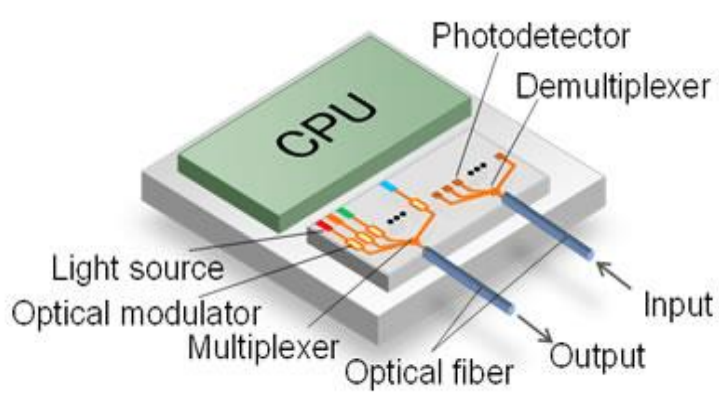

Figure 1. An Integrated Optical Transceiver for Optical Interconnect Aplication (5].

demultiplexer, and optical waveguides as the main important one. By using the prototype device, achieve optical modulation signals at speeds of $10 \mathrm{Gbps}$ at temperatures ranging from $25^{\circ} \mathrm{C}$ to $60^{\circ} \mathrm{C}$ can be obtained. Based on this, the optical interconnect can be used to carry out large data capacity.

Actually, the optical waveguides are very important in the integrated optical circuit and optical interconnect. They have several structure types such as circular and planar structure. The circular structure is applied to optical fiber cable [6]. The planar structure is usually used for optical components, integrated optic circuits, or optical interconnects [7]. Several methods for optical waveguide fabrications were reported using diffusion and ion exchange methods [8]-[10]. The methods have advantages each other, the optical waveguide propagation loss is one parameter to be considered furthermore. One candidate to realize the low propagation loss of optical waveguides is using silicon 
material for the optical waveguide since it has good performance in the propagation loss [11].

In this paper, we report design and analysis of the planar optical waveguides. In this paper, fabrication of planar optical waveguides using a polyimide material on thin silicon dioxide combined with the silicon substrate is also reported. The polyimide material is used for the core of the optical waveguides. The silicon dioxide located on a silicon substrate and the air is used for cladding of the optical waveguides. Fabrication of the optical waveguides such as oxidation, photoresist coating, masking, ultra-violet exposure, and etching was done. The fabricated optical waveguides were characterized physically using a standard microscope and scanning electron microscope (SEM). The fabrication processes and characterization results are reported in this paper.

\section{RESEARCH METHOD}

Figure 2 shows a structure of the polyimide optical waveguides fabricated on silicon dioxide combined with the silicon substrate. Refractive indices of the materials should be considered for guiding light. Commonly, the refractive index of the optical waveguide core must have larger than surrounding materials which are a substrate for optical waveguide cladding or cover [7][12]. In Figure 2 (a), a polyimide material with refractive index of 1.78 is used for optical waveguide core and a silicon dioxide layer with refractive index of 1.5 is used for optical waveguide cladding. Silicon material is used as a substrate for supporting the proposed device. Mode operation of optical waveguides can be designed by setting the core size. The optical waveguide core size is composed of width (W) and height $(\mathrm{H})$ values as shown in Figure $2(\mathrm{~b})$.

Analysis of the polyimide optical waveguide was done using Marcatilli's methods. Modal dispersion and electric field distribution for transfer-electric (TE) and transfer-magnetic (TM) modes were calculated and

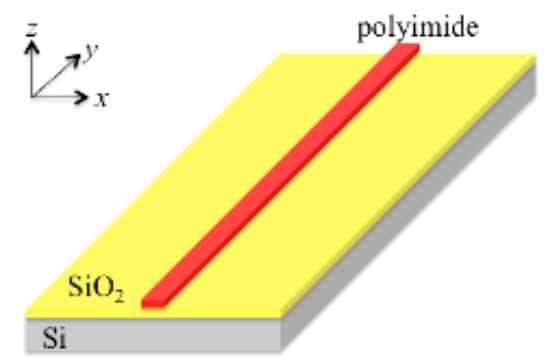

(a)

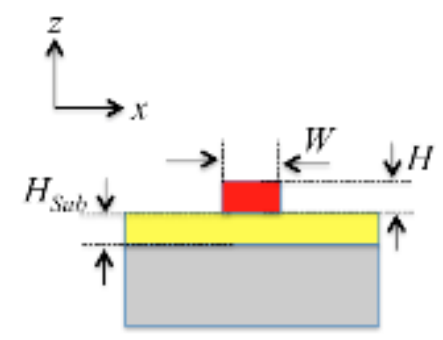

(b)

Figure 2. Structure of the Polyimide Optical Waveguides,

(a) The 3-D View, (b) Cross-Sectional View.
TABLE 1

PARAMETERS OF THE OPTICAL WAVEGUIDES

\begin{tabular}{|c|c|}
\hline Parameters & Value \\
\hline $\begin{array}{ll}\text { Optical waveguide core } \\
\text { - } & \text { Material } \\
\text { - } & \text { Height }(\mathrm{H}) \\
\text { - } & \text { Width }(\mathrm{W}) \text { for single mode } \\
\text { - } & \text { Width (W) for multi-mode }\end{array}$ & $\begin{array}{l}\text { Polyimide } \\
\sim 0.5 \mu \mathrm{m} \\
<1.6 \mu \mathrm{m} \\
>1.6 \mu \mathrm{m}\end{array}$ \\
\hline $\begin{array}{l}\text { Optical waveguide substrate } \\
\text { - } \quad \text { Material } \\
\text { - } \quad \text { Thickness }\end{array}$ & $\begin{array}{l}\text { Silicon Dioxide } \\
\sim 2 \mu \mathrm{m}\end{array}$ \\
\hline $\begin{array}{ll}\text { Supported substrate } \\
\text { - } & \text { Material } \\
\text { - } & \text { Thickness }\end{array}$ & $\begin{array}{l}\text { Silicon } \\
1 \mathrm{~mm}\end{array}$ \\
\hline
\end{tabular}

analyzed. By considering the calculated modal dispersion, the optical waveguides with certain mode operation, which are in single or multi modes, can be designed. Based on the reported paper, first, a slab optical waveguide in $\mathrm{z}$-axis was calculated for optical waveguide core height $(\mathrm{H})$ dependences. For single mode operation in $1550 \mathrm{~nm}$ optical wavelength, the core height must be below $0.5 \mu \mathrm{m}$ with effective refractive index of 1.65. When the optical waveguide core height is set over than the cut-off of single mode operation, the multi-mode operation will be induced. By setting core height of $0.5 \mu \mathrm{m}$, the modal dispersion for core width (W) dependence can be calculated. The core width of $1.6 \mu \mathrm{m}$ with effective refractive index of 1.63 was obtained for single mode operation. When the core size is over $0.5 \mu \mathrm{m}$ for core height and $1.6 \mu \mathrm{m}$ for core width, the optical waveguides are operated in the multimode.

Based on the calculation results, we are trying to a realization the polyimide optical waveguides. For fabrication, the designed parameters of the optical waveguides are shown in Table 1.

\section{Fabrication Process}

Fabrication processes and steps of the polyimide optical waveguides are illustrated in Figure 3. First, a silicon wafer with 3 inch-size was prepared and cleaned by removing unwanted organic materials on the silicon surface using standard cleaning 1 (RCA-1) and 2 (RCA2) as shown in Figure 4. In the RCA-1, pure water, $\mathrm{NH}_{4} \mathrm{OH}$ (ammonium hydroxide), and $\mathrm{H}_{2} \mathrm{O}_{2}$ (hydrogen peroxide) were mixed with a concentration of $5: 1: 1$, respectively and heated at $75^{\circ} \mathrm{C}$ for 10 minutes. In RCA2, pure water, $\mathrm{HCl}$ (Hydrogen Chloride), and $\mathrm{H}_{2} \mathrm{O}_{2}$ (Hydrogen Peroxide) were mixed with a concentration of $6: 1: 1$, respectively and heated at $75^{\circ} \mathrm{C}$ for 10 minutes. Then, oxidation process of the cleaned silicon was done for a few hours to obtaining about $2 \mu \mathrm{m}$-thick silicon dioxide. Therefore, the substrate was ready for next fabrication processes of polyimide optical waveguide.

The optical waveguides were fabricated using standard lithography and wet etching processes. First, the substrate was covered by adhesive promotor liquid using a spin coating with a rotation speed of $3500 \mathrm{rpm}$ for 20 seconds. The adhesive promoter layer is used for increasing the adhesion strength of polyimide to the 
substrate. Then polyimide material was deposited on the adhesive promotor layer using a spin coating with a rotation speed of several variations for 60 seconds. The polyimide layer was kept about 24 hours to obtain strong layer. The polyimide material layers with several thicknesses were obtained. Then, photoresist liquid was coated on the polyimide surface using spin coating machine with a rotation speed of $2500 \mathrm{rpm}$ for 30 seconds and heating for 180 seconds at $90^{\circ} \mathrm{C}$.

After that, the device was exposure using ultraviolet light for a few seconds with a mask pattern, where the mask pattern for several optical waveguide widths (w1 to w4) of 10, 20,30, and $50 \mu \mathrm{m}$ was prepared before by ordering from a company as shown in Figure 5. The material of the mask is plastic. The picture of the ultra-violet light exposure machine is shown in Figure 6. Then, the device was immersed in photoresist development liquid.

After that, the device was etched using Tetra methyl ammonium hydroxide $\left(\left(\mathrm{CH}_{3}\right)_{4} \mathrm{NOH}\right)$ for removing polyimide partly. Finally, photoresist removal was done. Device curing at $350^{\circ} \mathrm{C}$ for 30 minutes was also done on a hot plate. The polyimide optical waveguides were successfully fabricated on silicon dioxide stacked with the silicon substrate.

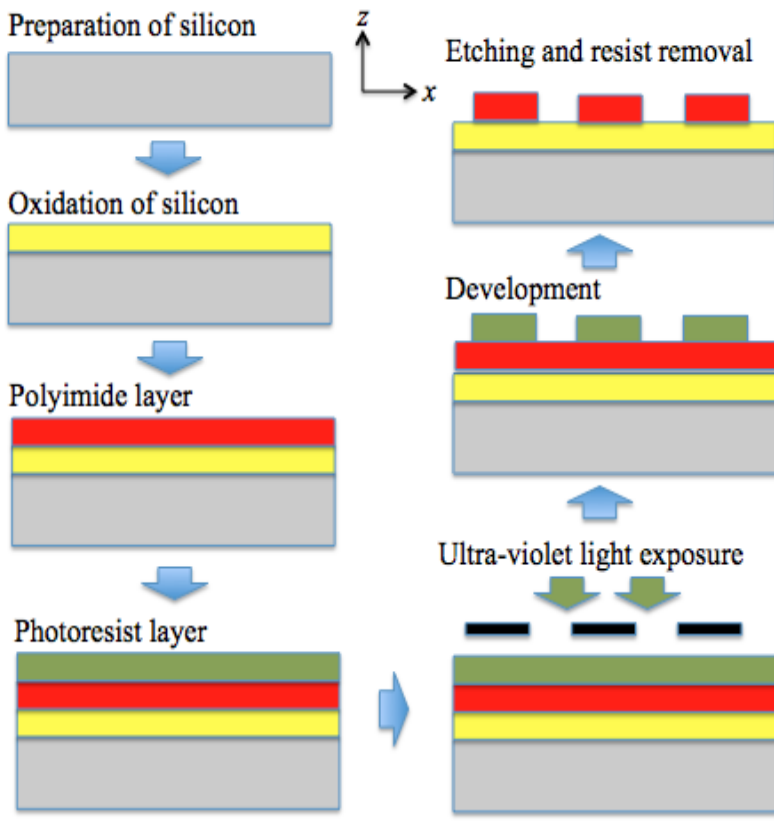

Figure 3. Fabrication Process and Steps of The Polyimide Optical Waveguides
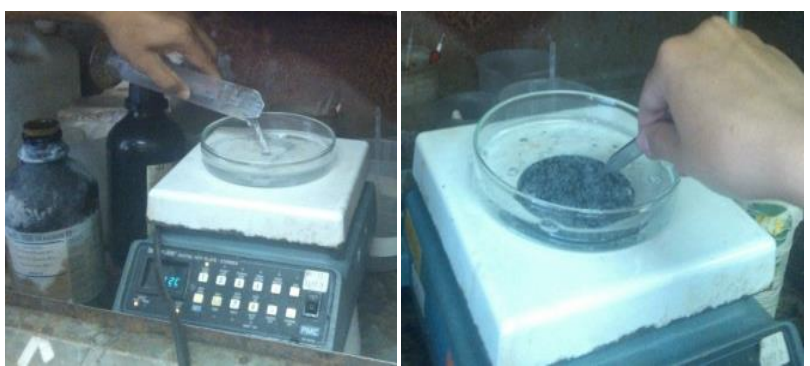

Figure 4. The Cleaning Process of Silicon Substrate using RCA-1 and RCA-2.

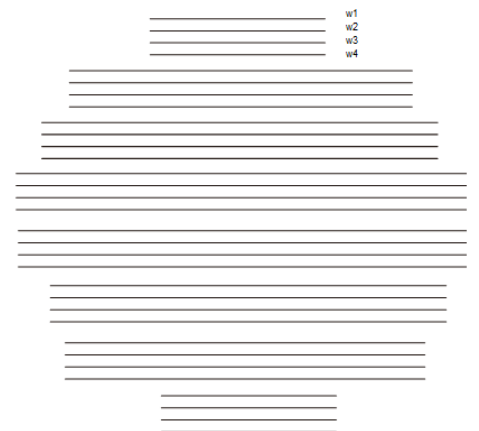

(a)

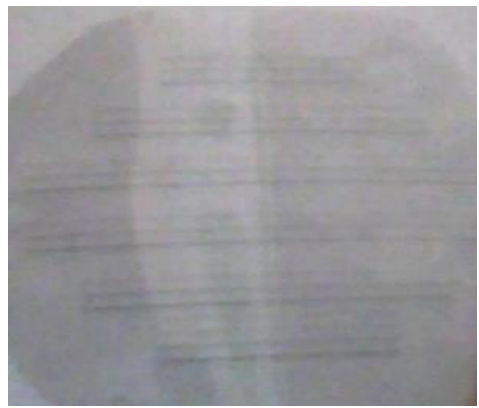

(b)

Figure 5. Mask of The Optical Waveguides (A) The Design and (b) The Real Mask.
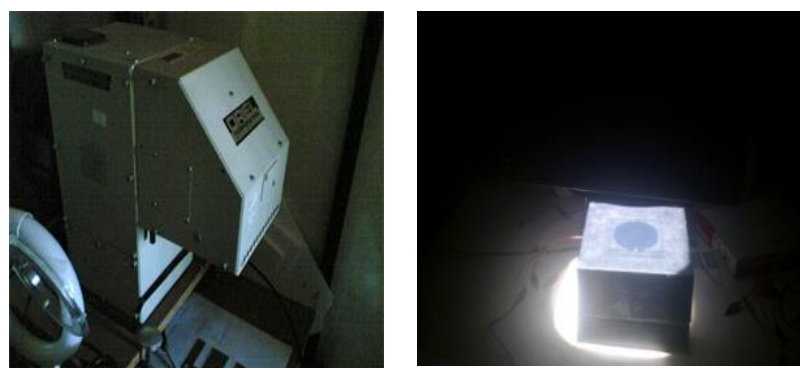

Figure 6. Ultra-violet Exposure Machine for Patterning Optical Waveguides.

\section{CHARACTERIZATIONS AND RESUlTS}

The polyimide optical waveguides were fabricated successfully. Photograph of the fabricated optical waveguides is shown in Figure 7. Optical waveguide thickness or polyimide height $(\mathrm{H})$ can be controlled using rotation speed during a spin coating process. The optical waveguide height was measured for several variations of spin coating speeds. The measured thickness $(\mathrm{H})$ of the fabricated optical waveguides are shown in Figure 8. We can see that thin optical waveguide can be obtained using faster spin coating speed.

We have measured the optical waveguides using profile meter as shown in Figure 9. Clear patterns of optical waveguides can be observed. The optical waveguides are quite small, the optical waveguides can be observed clearly using the profile meter. Measurement results of the optical waveguide width are shown by green-bar in Figure 10. The fabricated optical waveguides have fabrication error about $6-8 \mu \mathrm{m}$ larger than pattern widths in the mask as shown by purple-bar in Figure 10. Fabrication errors might be due to the distance between mask and device during ultra-violet light exposure, exposure time with ultra-violet light, 
time of development process, and time of etching process. Therefore, the fabricated optical waveguides become wide.

In order to measure the optical waveguides in detail, the standard microscope was used (Figure 11). The optical waveguide width can be measured in detail. The measured optical waveguide width is shown by orange-bar in Figure 10. The fabricated optical waveguides have fabrication error almost negligible in part.

The fabricated optical waveguides were also observed using scanning electron microscope (SEM). Photographs of the measured optical waveguide using SEM are shown in Figure 12. Figure 12 (a) shows the cross-sectional view of the fabricated optical waveguides. Clear optical waveguide core, which is polyimide, can be observed. Figure 12 (b) shows the top view of the fabricated optical waveguides for measuring the width. The measured width is shown by red-bar in Figure 10. The measurement results using SEM have good accuracy. An error of optical waveguide width is induced, it might be due to during fabrication process. Therefore, several treatments in the fabrication process should be considered carefully.

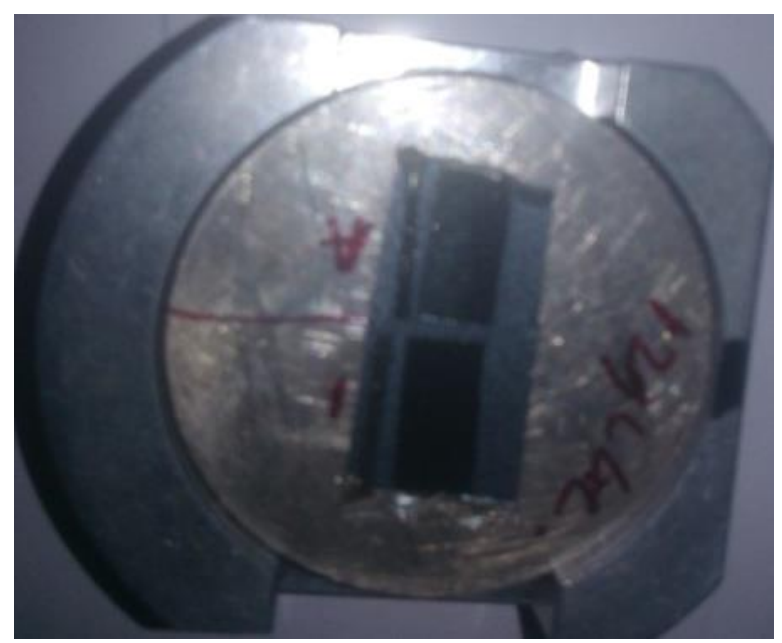

Figure 7. Photograph of The Fabricated Optical Waveguides

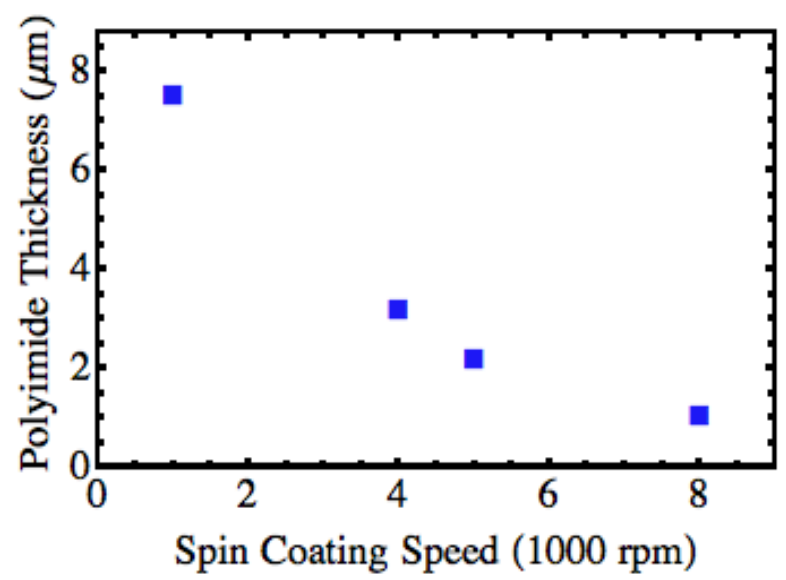

Figure 8. Measured Polyimide Thickness for Several Variations of Spin Coating Speeds
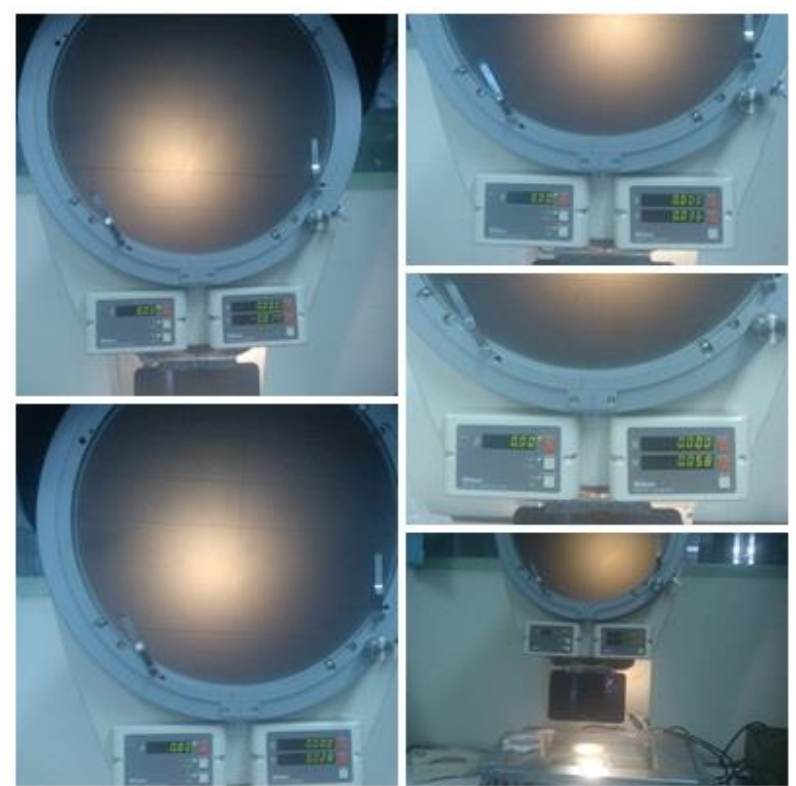

Figure 9. Observation of Optical Waveguide using Profile Meter.

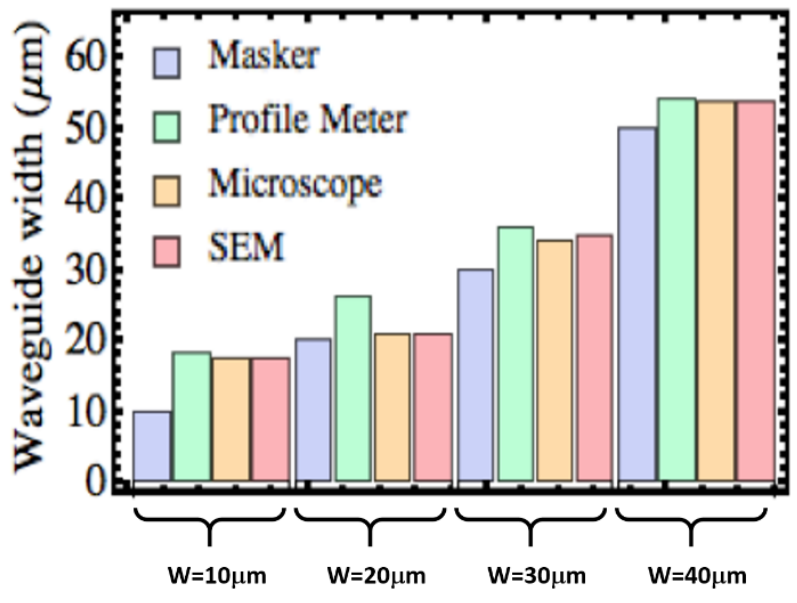

Figure 10. Measured Width of The Fabricated Optical Waveguides.
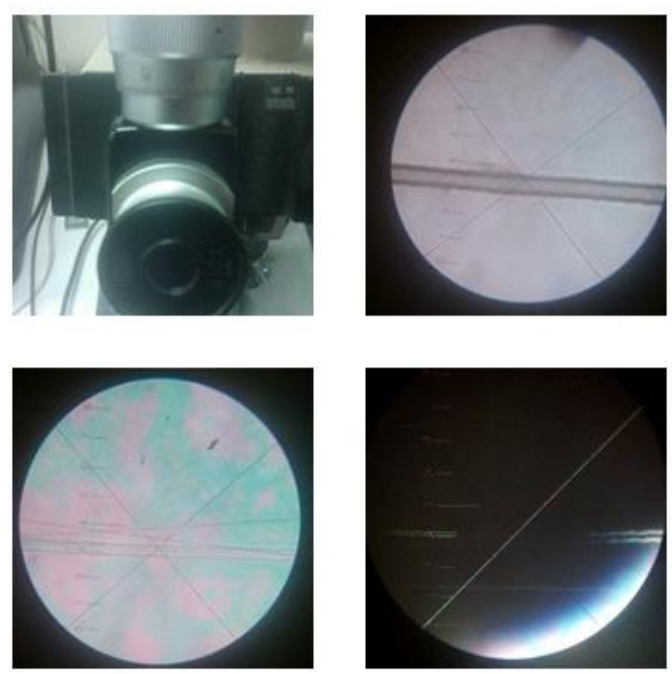

Figure 11. Measurement of An Optical Waveguide using A Standard Microscope. 


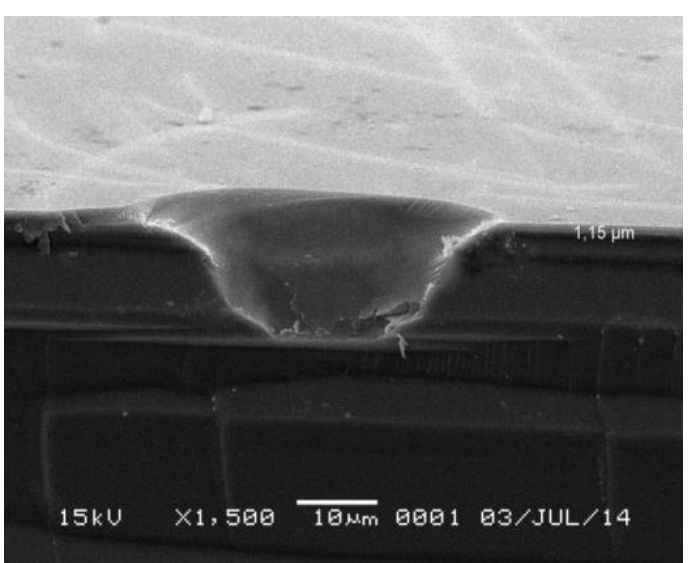

(a)
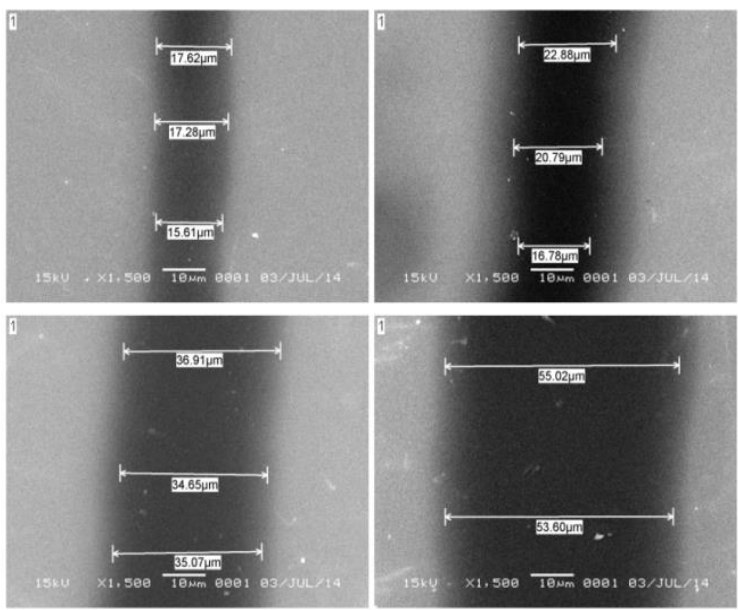

(b)

Figure 12. Measurement of Optical Waveguides using Sem: (A) Cross-Sectional View and (B) Top View for Measuring The Width.

\section{CONCLUSION}

We have reported fabrication and characterization of planar optical waveguides using a polyimide material on thin silicon dioxide combined with the silicon substrate. The polyimide material is used for the core of the optical waveguides. The silicon dioxide located on the silicon substrate and the air is used for cladding of the optical waveguides. Fabrication of the optical waveguides such as oxidation, photoresist coating, masking, ultraviolet exposure, and etching was done. The fabricated optical waveguides were characterized physically using a standard microscope and scanning electron microscope (SEM). The fabrication processes and characterization results were also reported. Promising applications of the polyimide optical waveguides are also discussed.

The fabricated optical waveguide can be used for transmitting light wave especially for an integrated optical circuit in future high-speed data transfer, interconnects, and sensing applications [13]-[14]. We believe that the high-speed communication is required in the future to carry-out high capacity data from one place to other places. Additionally, it can be used also for signal processing unit based on an optical wave [15]-[16].

\section{ACKNOWLEDGEMENT}

The authors would like thanks to Dr. G. Sugandi, Dr. Dedi, and Mr. I. D. P. Hermida from Research Center for Electronics and Telecommunication, Indonesian Institute of Sciences (LIPI) for their kind supports and help during the experiment of the device fabrication and measurement in the laboratory and valuable comments and suggestions during the interactive discussion.

This research activity is financially supported in partly Indonesian Institute of Sciences (LIPI) Indonesia through the competitive projects with the title of "Development of Optical Environmental Sensors" and the "Research and Development of Radio and Optical Components Based-on Planar Structure for Communication and Sensing".

\section{REFERENCE}

[1] M. Cvijetic, I. B. Djordjevic, Advanced Optical Communication Systems and Networks, Norwood, USA: Artech House, 2013.

[2] S. Iezekiel, Microwave Photonic: Device and Applications, Chichester, UK: John Wiley \& Sons, Ltd., 2009.

[3] Y. N. Wijayanto, A. Kanno, H. Murata, T. Kawanishi, and Y. Okamura, "Millimeter-wave radar receiver using Z-cut LiNbO3 optical modulator with orthogonal-gap-embedded patchantennas on low-k dielectric material," IEICE Trans. on Electron., vol. E98-C, no. 8, pp. 783-792, August 2015.

[4] B. Mulyanti, P. M. Menon, S. Shaari, T. Hariyadi, L. Hasanah, H. Hroon, A. A. Ehsan, D. Mahmudin, G. Wiranto, B. Y. Majlis, "Design and optimization of coupled microring resonators in silicon-on-insulator," Sains Malay., vol. 43, no 2, pp. 247-252, 2014

[5] (2012) Integrated silicon optical transmitter to carry large volumes of data between CPUs. [Online]. Available: https://phys.org/news/2012-08-silicon-optical-transmitter-largevolumes.html

[6] M. F. M. Salleh, Z. Zakaria, "Optical fiber bending detection on long distance OPGW using OTDR”, Telkomnika, vol. 13, no. 13 2015 .

[7] K. Okamoto, Fundamental of Optical Waveguides, San Diego, USA: Academic Press (Elsevier), 2006.

[8] W. H. Huang and W. S. Wang, "Gallium in-diffusion for the fabrication of lithium niobate optical waveguides," in IEEE Photon. Technol. Lett., vol. 19, no. 20, pp. 1679-1681, Oct. 2007.

[9] S. Y. Gang, A. B. Mohammad, N. M. Kassim and M. H. Ibrahim, "Fabrication of polymeric optical waveguides," in Proc. 2004 RF and Microwave Conference (IEEE Cat No.04EX924), 2004, pp. 265-268.

[10] Y. N. Wijayanto, D. Mahmudin, and P. Daud, "Design of rectangular optical waveguide on $\mathrm{LiTaO} 3$ crystal using thermal annealed proton exchange methods," Jurnal Elektronika dan Telekomunikasi, vol. 14, no. 1, pp. 20-23, Juni 2014.

[11] D. Mahmudin and Y. N. Wijayanto, "Optical waveguide using polymer material on silicon dioxide substrate for $1,55 \mu \mathrm{m}$ optical wavelength," Jurnal Elektronika dan Telekomunikasi, vol. 14, no. 2, pp.56-60, Des. 2014

[12] P. W. Juodawlkis, J. J. Plant, F. J. O'Donnell and J. B. Schlager, "Slab-coupled optical waveguide devices for low-noise signal generation," in Proc. 2007 Digest of the IEEE/LEOS Summer Topical Meetings, Portland, OR, 2007, pp. 167-168.

[13] X. Xu, L. Ma, W. Zhang, J. Du and Z. He, "Fabrication and performance analysis of polymer waveguides for optical interconnects," in Proc. 2016 IEEE Optical Interconnects Conference (OI), San Diego, CA, 2016, pp. 76-77.

[14] A. Kanno, T. Umezawa, T. Kuri, N. Yamamoto, T. Kawanishi, Y. N. Wijayanto, "Key technologies for millimeter-wave distributed radar system over a radio over fiber network," in Proc. 2016 International Conference on Radar, Antenna, Microwave, Electronics, and Telecommunications, Tangerang, Indonesia, 2016

[15] M. Galili, F. Da Ros, H. Hu, M. Pu, K. Yvind and L.K. Oxenløwe, "Ultra-broadband optical signal processing using 
AlGaAs-OI devices," in Proc. 2017 Optical Fiber Communications Conference and Exhibition (OFC), Los Angeles, CA, 2017, pp. 1-3.
[16] G. P. Agrawal, "Optical signal processing," in Fiber-Optic Communication Systems, $4^{\text {th }}$ ed., New York, USA: John Wiley \& Sons, Inc., 2012 